\title{
Quantum Phase Transition for One-Dimensional Spin-1 XXZ Model with Uniaxial Single-Ion-Type Anisotropy
}

\author{
Chunhuan Xiang1, Honglei Wang2* \\ ${ }^{1}$ School of Public Health and Management, Chongqing Medical University, Chongqing, China \\ ${ }^{2}$ College of Medical Informatics, Chongqing Medical University, Chongqing, China \\ Email: *w8259300@163.com
}

How to cite this paper: Xiang, C.H. and Wang, H.L. (2019) Quantum Phase Transition for One-Dimensional Spin-1 XXZ Model with Uniaxial Single-Ion-Type Anisotropy. Journal of Applied Mathematics and Physics, 7, 1513-1518.

https://doi.org/10.4236/jamp.2019.77102

Received: June 10, 2019

Accepted: July 19, 2019

Published: July 22, 2019

Copyright () 2019 by author(s) and Scientific Research Publishing Inc. This work is licensed under the Creative Commons Attribution International License (CC BY 4.0).

http://creativecommons.org/licenses/by/4.0/

\begin{abstract}
The quantum phase transitions for one-dimensional spin-1 XXZ model with uniaxial single-ion-type anisotropy are investigated by numerical simulation. The Gaussion transition occurred between the Larged- $D$ phase and Neel phase, which is a gapped phase. The results of ground state energy, local order parameter and entanglement entropy agree with each other. The figures for all of the physical observable are shown in the text.
\end{abstract}

\section{Keywords}

Local Order Parameter, Phase Diagram, Entanglement Entropy, Ground State Energy

\section{Introduction}

Investigation on the properties of quantum phase transition [1] [2] [3] [4] [5], which occurs as a result of a sudden change in the ground state but the outside control parameter changed slowly, for the one-dimensional spin-1 Heisenberg systems is going on through the last several decades. For the quantum system, quantum fluctuations instead of thermal fluctuations, drive the quantum phase transitions in absolute zero temperature. Meanwhile, Haldane [6] [7] [8] [9] suggested that a gapless ground state appeared for half integer spin; there is a gap between the first excited state and the ground state for integer spin.

The one-dimensional spin-1 XXZ model is an important model and had been researched by many groups [10] [11] [12] [13], which is destroyed by various types of perturbations: uniaxial single-ion-type anisotropy, bond alternation. The critical point in the thermodynamic limit is still difficult to obtain. On the 
other hand, G. Vidal and his colleagues had introduced the matrix product states [14] [15] [16] [17] [18] in one spatial dimension for infinite lattice in the thermodynamic limit, which is based on the variational algorithm.

The good approximation ground-state wave-function can be obtained with the given initial state.

This paper is organized as follows. In the next section, the model Hamiltonian is given and the phase diagram is shown. The physical observable for the model are explained in Section 3. The final section is devoted to a summary.

\section{The Hamiltonian and Ground-State Phase Diagram}

The Hamiltonian for the one dimensional spin-1 XXZ model with uniaxial single-ion-type anisotropy [10] is given as the follow

$$
H=\sum_{l=1}^{N}\left[J\left(S_{l}^{x} S_{l+1}^{x}+S_{l}^{y} S_{l+1}^{y}\right)+J_{z} S_{l}^{z} S_{l+1}^{z}\right]+D \sum_{l=1}^{N}\left(S_{l}^{z}\right)^{2}
$$

where $J$ and $J_{z}$ are the exchange couplings for $x, y$ directions and $z$-direction, respectively, $D$ is the external magnetic field, and $S$ is the spin- 1 operator on the site $l$.

$$
S^{x}=\frac{1}{\sqrt{2}}\left(\begin{array}{ccc}
0 & 1 & 0 \\
1 & 0 & 1 \\
0 & 1 & 0
\end{array}\right), \quad S^{y}=\frac{1}{\sqrt{2}}\left(\begin{array}{ccc}
0 & -\mathrm{i} & 0 \\
\mathrm{i} & 0 & -\mathrm{i} \\
0 & \mathrm{i} & 0
\end{array}\right), \quad S^{z}=\left(\begin{array}{ccc}
1 & 0 & 0 \\
0 & 0 & 0 \\
0 & 0 & -1
\end{array}\right)
$$

With the exchange couplings $D=0$ and $J_{z}=1$, Equation (1) is deduced into one-dimensional quantum Heisenberg model. The phase diagram for Equation (1) is shown in Figure 1. The Large- $D$, Neel, XY, Haldane, Ferromagnetic phase are appeared in the diagram with $J_{z}$ and $D$ as the control parameter. Gaussian phase transition happened between the Haldane phase and Large- $D$ phase, which is a gapful phase to gapful phase. An Ising transition occurred between

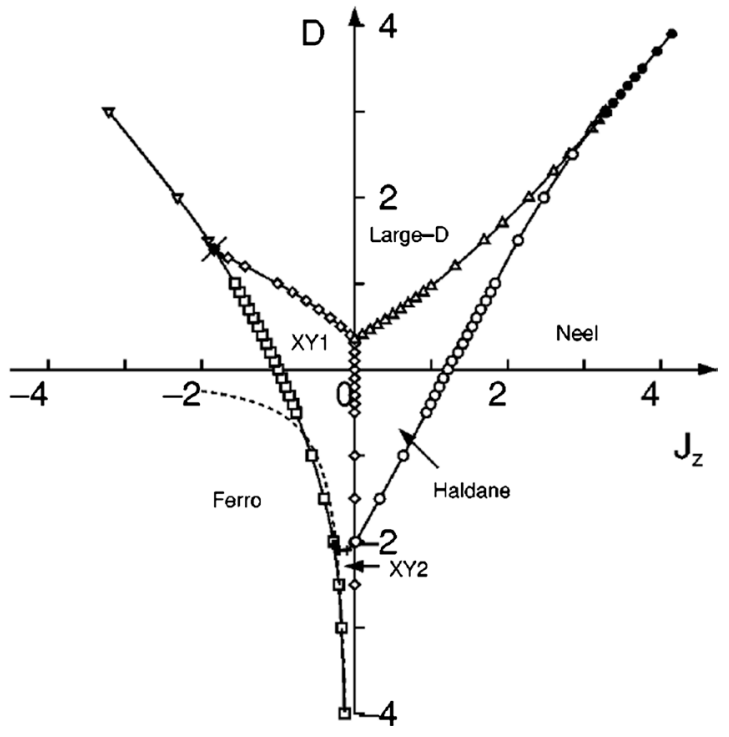

Figure 1. Ground state phase diagram [10] of the Hamiltonian (1). The Large- $D$ phase, Neel phase, Haldane phase, two XY phase and Ferromagnetic phase are shown with different label. $J_{z}$ and $D$ are the control parameter in $x$ and $y$ direction. 
the Neel phase and Haldane phase. In this paper, we set $J=1, J_{z}=5$ and $10, D$ as the out control parameter. The phase transition between the Neel phase and Large- $D$ phase is studied from the ground state energy, local order parameter and the entanglement entropy.

\section{Physical Observable}

When the out control parameter changed, the phase transition will occurred for the Hamiltonian Equation (1). The physical observable is different behavior in different phase, which changed obviously in the critical point for the given truncation dimension. The Hilbert space is labeled as $d$ (physical space), the truncation dimension is the auxiliary space in the matrix product states. By using transitionally invariant of quantum systems on an infinite-size lattice, the critical point is arose, which is near and near the critical point in the thermodynamic limit with the truncation dimension larger and larger. The ground energy (Figure 2), local order parameter and entanglement entropy are shown for the Large- $D$ phase and Neel phase with different truncation dimension.

The local order parameter is an important observable in phase transition field, which is obtained by order parameter and the good approximation ground state wavefunction. The order parameter can be read from the two-site reduced density matrix. The spin-1 $S_{z}$ is the order parameter. The simulation results of the local order parameter for Equation (1) are shown in Figure 3 with truncation dimension $\chi=8,16,32$ and 50 with different label in left $\left(J_{z}=5\right)$ and right $\left(J_{z}=\right.$ 10), respectively. The jump of the local order parameters shown the type of the phase transition between the Large- $D$ phase and the Neel phase is the first phase transition. The lower local order parameter appears in the critical point with the parameter $J_{z}=5$ (the left figure). The jump of order parameter will lower and lower with the smaller and smaller $J_{z}$. The tri-critical point among the Large- $D$
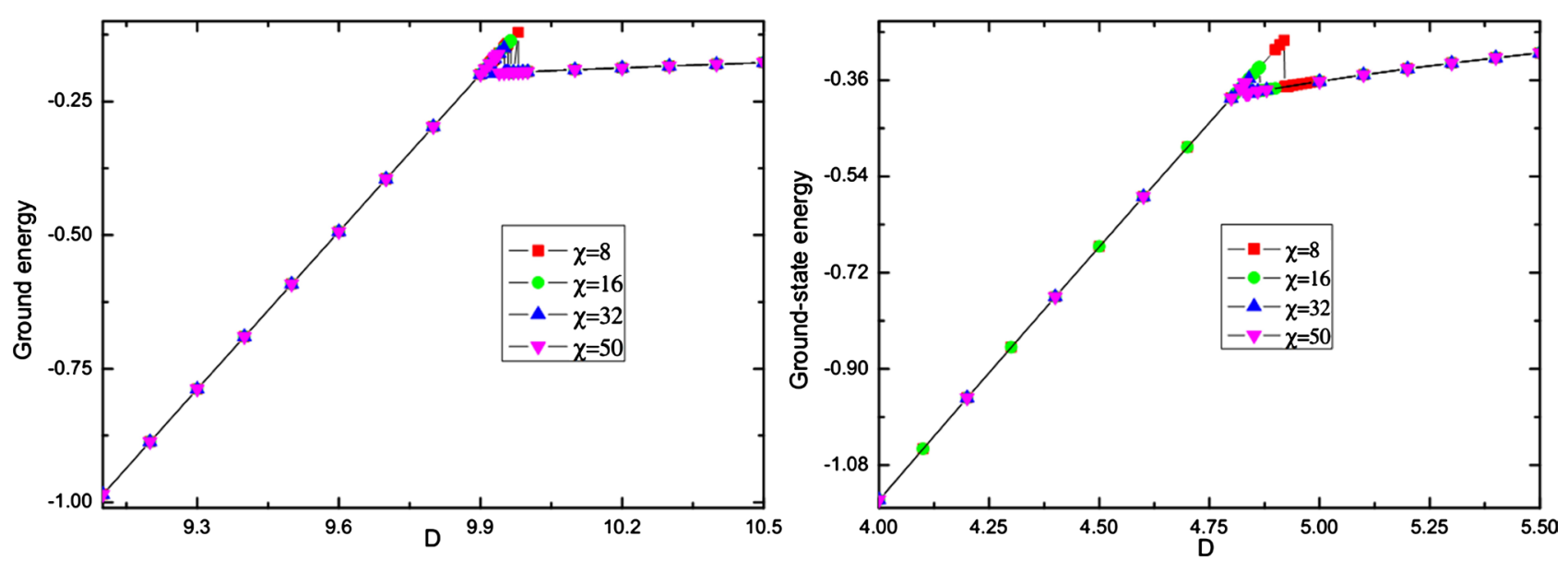

Figure 2. The ground state energy for the Hamiltonian (1) with $J=1, J_{z}=5$ (left) and $J_{z}=10$ (right). The truncation dimension is selected as $\chi=8,16,32,50$ with different label in the figure. The figures tell us that the ground state energy in the Neel phase is lower than the one in the Large- $D$ phase. A jump appears in the critical point given by the corresponding truncation. The height of the jump with $J_{z}=10$ is larger than the jump with $J_{z}=5$. The jump means that the first phase transition occurred between the Larged- $D$ phase and Neel phase. 

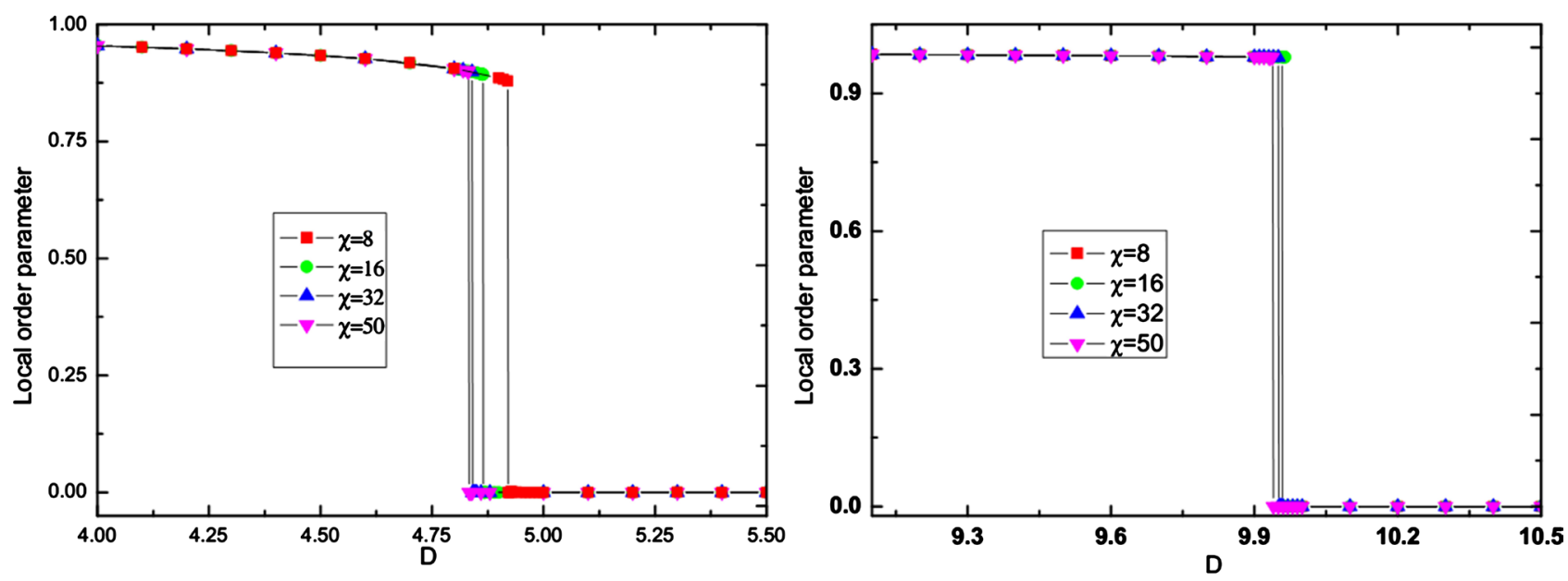

Figure 3. The local order parameter for the Hamiltonian (1) with $J=1, J_{z}=5$ (left) and 10 (right), $D$ as the out control parameter, the truncation dimensions are shown with $\chi=8,16,32,50$ and different label. The jumps appear in both figures, which tell us that the type of the phase transition is the first phase transition. The jump in the phase transition point of the left is lower than the right one.

phase, Neel phase and Haldane phase arise. The corresponding type of the phase transition is changed into second phase transition.

The order or disorder can be quantified in terms of the von Neumann entropy, which is used to descript the amount of entanglement captured between two half infinite chains. The von Neumann entropy $S$ for half infinite chain $(A)$ or half infinite chain $(B)$ is defined as

$$
S=-\operatorname{Tr} \rho_{A} \log \rho_{A}=-\operatorname{Tr} \rho_{B} \log \rho_{B}
$$

where $\rho_{A(B)}$ is the reduced density matrix. The fluctuation is trivial outside the phase transition point, however, which become stronger and stronger with the control parameter near and near the phase transition point. The fluctuation is strong enough to destroy the order completely. The entanglement entropy is divergence in the phase transition point in theory. The entanglement entropy for the Hamiltonian (1) with $J=1, J_{z}=5$ (left) and 10 (right) is shown in Figure 4 with the truncation dimension $\chi=8,16,32,50$ in different label.

The jump appears in the entanglement entropy, which means the type of the transition is the first phase transition. The shift of the phase transition line is smaller and smaller with the truncation dimension is bigger and bigger. The figures tell us that the truncation dimension is larger enough to capture the amount of the entanglement entropy.

\section{Summary}

The one-dimensional spin-1 XXZ model with uniaxial single-ion-type anisotropy is investigated by using matrix product states. The ground state energy, local order parameter and the entanglement entropy for the model with $J=1, J_{z}=5$ and 10 , and the parameter $D$ as the out control parameter are shown in this paper. The jump in physical observable for $J_{z}=10$ is larger than the one with parameter for $J_{z}=5$. The jump tells us that the phase transition between the Large- $D$ phase and 

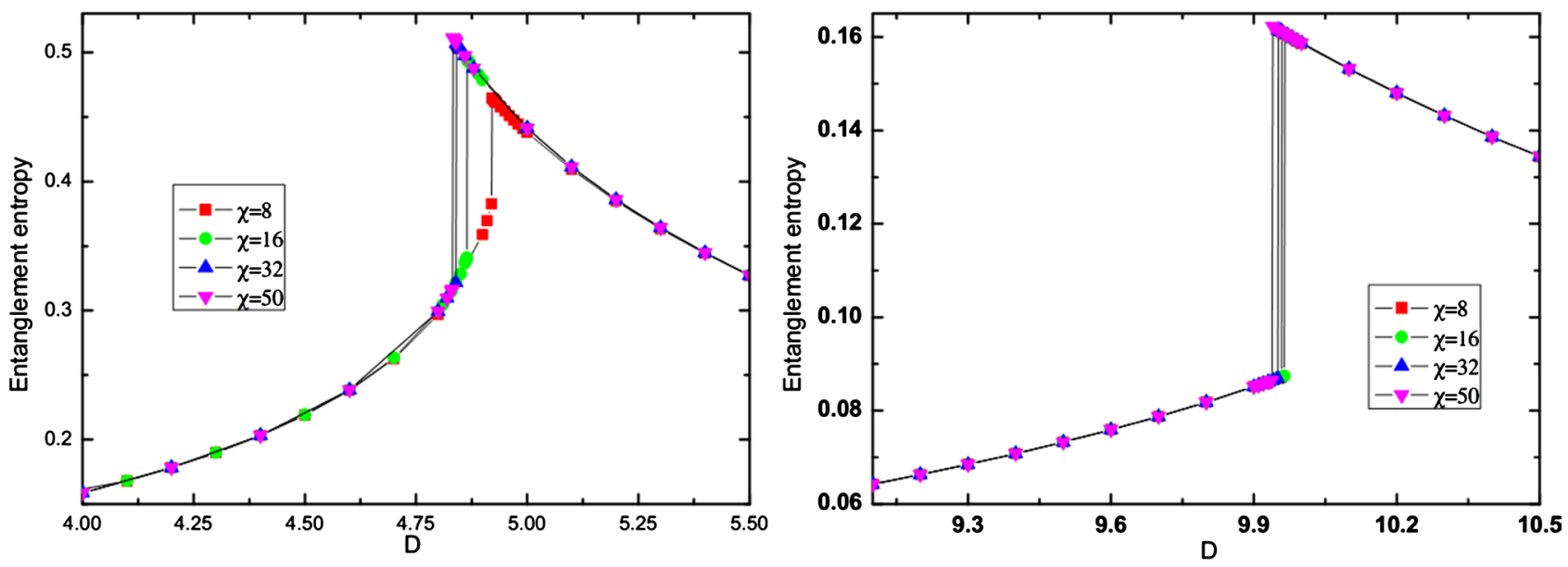

Figure 4. The entanglement entropy of the Hamiltonian (1) with truncation dimension $\chi=8,16,32,50$ with different label, the parameters are given as $J=1, J_{z}=5$ (left) and 10 (right). $D$ as the out control parameter.

Neel phase is the first phase transition. As the $J_{z}$ is smaller and smaller, the jump will lower and lower. The tricritical point appears, which happened among the Large- $D$ phase, Neel phase and Haldane phase. The jump disappear thoroughly. All the results obtained from the physical observables agree well with each other.

\section{Conflicts of Interest}

The authors declare no conflicts of interest regarding the publication of this paper.

\section{References}

[1] Sachdev, S. (2013) Quantum Phase Transitions. Cambridge Univ. Press, Cambridge.

[2] Langen, T., Geiger, R., Kuhnert, M., Rauer, B. and Schmiedmayera, J. (2013) Dynamical Phase Transition in the 1D-Transverse Field Ising Chain Characterized by the Transverse Magnetization Spectral Function. Nature Physics, 9, 640-643. https://doi.org/10.1038/nphys2739

[3] Langen, T., Erne, S., Geiger, R., Rauer, B., Schweigler, T., Kuhnert, M., Rohringer, W., Mazets, I., Gasenzer, T. and Schmiedmayer, J. (2015) The Principle of Antagonism Ensures Protein Targeting Specificity at the Endoplasmic Reticulum. Science, 348, 201-207. https://doi.org/10.1126/science.1257026

[4] Polkovnikov, A., Sengupta, K., Silva, A. and Vengalattore, M. (2011) Colloquium: Nonequilibrium Dynamics of Closed Interacting Quantum Systems. Reviews of Modern Physics, 83, 863. https://doi.org/10.1103/RevModPhys.83.863

[5] Gogolin, C. and Eisert, J. (2016) Equilibration, Thermalisation, and the Emergence of Statistical Mechanics in Closed Quantum Systems. Reports on Progress in Physics, 79, Article ID: 056001. https://doi.org/10.1088/0034-4885/79/5/056001

[6] Haldane, F.D.M. (1983) Nonlinear Field Theory of Large-Spin Heisenberg Antiferromagnets: Semiclassically Quantized Solitons of the One-Dimensional Easy-Axis Néel State. Physical Review Letters, 50, 1153-1156. https://doi.org/10.1103/PhysRevLett.50.1153

[7] Haldane, F.D.M. (1983) Continuum Dynamics of the 1-D Heisenberg Antiferromagnet: Identification with the $\mathrm{O}(3)$ Nonlinear Sigma Model. Physics Letters A, 93, 
464-468. https://doi.org/10.1016/0375-9601(83)90631-X

[8] Glaus, U. and Schneider, T. (1984) Critical Properties of the Spin-1 Heisenberg Chain with Uniaxial Anisotropy. Physical Review B, 30, 215-225.

https://doi.org/10.1103/PhysRevB.30.215

[9] Chen, W., Hida, K. and Nakano, H. (1999) Magnetization Plateaus in One Dimensional S $=1 / 2$ Heisenberg Model with Dimerization and Quadrumerization. Journal of the Physical Society of Japan, 68, 625-630.

[10] Chen, W., Hida, K. and Sanctuary, B.C. (2003) Ground-State Phase Diagram of S-1 XXZ Chains with Uniaxial Single-Ion-Type Anisotropy. Physical Review B, 67, Article ID: 104401. https://doi.org/10.1103/PhysRevB.67.104401

[11] Tasak, H. (1991) Quantum Liquid in Antiferromagnetic Chains: A Stochastic Geometric Approach to the Haldane Gap. Physical Review Letters, 66, 798-801. https://doi.org/10.1103/PhysRevLett.66.798

[12] Schulz, H.-J. (1986) Phase Diagrams and Correlation Exponents for Quantum Spin Chains of Arbitrary Spin Quantum Number. Physical Review B, 34, 6372-6385. https://doi.org/10.1103/PhysRevB.34.6372

[13] den Nijs, M. and Rommelse, K. (1989) Preroughening Transitions in Crystal Surfaces and Valence-Bond Phases in Quantum Spin Chains. Physical Review B, 40, 4709-4734. https://doi.org/10.1103/PhysRevB.40.4709

[14] Fannes, M., Nachtergaele, B. and Werner, R.F. (1992) Finitely Correlated States on Quantum Spin Chains. Communications in Mathematical Physics, 144, 443-490. https://doi.org/10.1007/BF02099178

[15] Ostlund, S. and Rommer, S. (1995) Thermodynamic Limit of Density Matrix Renormalization. Physical Review Letters, 75, 3537-3540. https://doi.org/10.1103/PhysRevLett.75.3537

[16] Perez-Garcia, D., Verstraete, F., Wolf, M.M. and Cirac, J.I. (2007) Matrix Product State Representations. Quantum Information and Computation, 7, 401-430.

[17] Verstraete, F., Porras, D. and Cirac, J.I. (2004) Density Matrix Renormalization Group and Periodic Boundary Conditions: A Quantum Information Perspective. Physical Review Letters, 93, Article ID: 227205. https://doi.org/10.1103/PhysRevLett.93.227205

[18] Vidal, G. (2007) Classical Simulation of Infinite-Size Quantum Lattice Systems in One Spatial Dimension. Physical Review Letters, 98, Article ID: 070201. https://doi.org/10.1103/PhysRevLett.98.070201 